\title{
Comparison of pseudo-living character of RAFT polymerizations conducted under homogeneous and heterogeneous conditions $\uparrow$
}

\begin{abstract}
Mona Semsarilar, Elizabeth R. Jones and Steven P. Armes*
RAFT dispersion polymerization of 2,2,2-trifluoroethyl methacrylate (TFEMA) is conducted in ethanol at $70^{\circ} \mathrm{C}$ using either poly(2-(dimethylamino)ethyl methacrylate) or poly(methacrylic acid) as a macromolecular chain transfer agent. If the diblock copolymer nanoparticles are not too large, the small refractive index difference between the PTFEMA cores and ethanol leads to minimal light scattering. This enables the pseudo-living character of RAFT formulations conducted under solution and dispersion polymerization conditions to be compared by monitoring the loss of RAFT chain-ends via UV-visible absorption spectroscopy. Significantly fewer chain-ends are lost during RAFT dispersion polymerization, suggesting that such heterogeneous formulations have greater pseudo-living character. Moreover, ${ }^{19} \mathrm{~F}$ NMR spectroscopy provides the first direct experimental evidence that RAFT dispersion polymerization proceeds via monomer-swollen block copolymer micelles. The relatively low refractive index of PTFEMA complicates GPC analysis, leading to apparent contamination of the diblock copolymer and erroneously high polydispersities. However, this artefact can be corrected by deconvolution of the GPC curves, followed by their reconstruction using appropriate refractive indices.
\end{abstract}

Received 2nd August 2013

Accepted 23rd August 2013

DOI: $10.1039 / c 3 p y 01042 b$

www.rsc.org/polymers

\section{Introduction}

Self-assembly of an $\mathrm{AB}$ diblock copolymer is driven by the mutual enthalpic incompatibility of the A and B blocks and is well known in both the solid state ${ }^{1,2}$ and in solution. ${ }^{3,4}$ In the latter case, a remarkable range of copolymer morphologies have been reported, including spheres, ${ }^{5}$ worms $/$ rods,${ }^{6-9}$ toroids, ${ }^{10}$ disks $^{11}$ and vesicles..$^{1,5,12-14}$ However, a significant disadvantage is that such nano-structures are usually generated via various post-polymerization processing routes, such as a solvent switch or thin film rehydration. ${ }^{15}$ However, these protocols are almost invariably conducted in dilute solution and therefore are not readily amenable to scale-up for the many potential applications suggested for block copolymer nanoparticles, which include drug delivery, colloidal templates, catalyst supports or biocompatible gels for cell storage and growth..$^{14,16-19}$

Over the last five years or so, there has been growing interest in polymerization-induced self-assembly (PISA) as a convenient means of generating the desired block copolymer morphology without requiring any post-polymerization processing step..$^{20-32}$

Department of Chemistry, The University of Sheffield, Brook Hill, Sheffield, Yorkshire, S3 7HF, UK. E-mail: s.p.armes@sheffield.ac.uk

$\dagger$ Electronic supplementary information (ESI) available: Further kinetic and GPC data; additional UV-visible spectra; digital photographs of the visual appearance of the final RAFT polymerization reaction mixtures; additional table summarizing RAFT dispersion and solution polymerization data. Excel spreadsheet for GPC deconvolution analysis. See DOI: 10.1039/c3py01042b
PISA syntheses offer several important advantages over traditional solution polymerizations. For example, relatively fast rates of polymerization are often observed because once micellar nucleation occurs at some intermediate conversion, the unreacted monomer is preferentially solubilized within the micelles as a cosolvent for the growing core-forming block, which leads to a faster rate of polymerization. ${ }^{23,24,29,33}$ However, although this hypothesis is consistent with various experimental observations, no direct experimental evidence has been offered to support it. A second important advantage for PISA formulations is that good control over the solution viscosity is possible because the block copolymer is generated in the form of sterically-stabilized nanoparticles, rather than molecularly dissolved copolymer chains. Thirdly, various desired block copolymer morphologies (e.g. spheres, worms or vesicles) can be generated directly in relatively concentrated solution (up to $25-30 \%$ solids). ${ }^{23-26,28-30,34-39}$ Charleux and co-workers have used various water-immiscible monomers such as styrene, $n$-butyl acrylate or methyl methacrylate to generate the hydrophobic block in their RAFT aqueous emulsion polymerization formulations. ${ }^{34-39}$ Appropriate selection of the core-forming block for a given solvent is arguably more stringent for RAFT dispersion polymerization formulations, since the vinyl monomer should be miscible with the continuous phase, while the corresponding polymer must be insoluble. Various thermo-responsive polymers have been utilized for aqueous PISA formulations, including poly( $N$-isopropylacrylamide), poly $\left(N, N^{\prime}\right.$-diethyl acrylamide), poly(2-hydroxypropyl methacrylate) and poly(2-methoxyethyl acrylate). ${ }^{20,21,23-25,27,29-31,34,35,39-42}$ 
For PISA syntheses conducted in alcoholic solvents, benzyl methacrylate has been $\operatorname{shown}^{26,28,43}$ to offer a significantly enhanced rate of polymerization compared to styrene; ${ }^{44-47}$ selection of the former monomer allows much higher final conversions to be achieved within reasonable time scales. Charleux's group has also reported the use of a cholesterolbased vinyl monomer, which allows greater access to the elusive worm phase. ${ }^{38}$

In the present work, we report the first example of a semifluorinated monomer, 2,2,2-trifluoroethyl methacrylate (TFEMA), as the core-forming block for RAFT dispersion polymerizations conducted in ethanol. TFEMA is utilized in various commercial applications because of its relatively low refractive index. ${ }^{48-51}$ As we shall see, this particular monomer/solvent combination offers a unique opportunity to perform comparative experiments that shed new light on how RAFT dispersion polymerizations differ from conventional RAFT solution polymerizations.

\section{Results and discussion}

In PISA syntheses, spheres, worm-like micelles or vesicles can be readily prepared using either emulsion or dispersion polymerization formulations in combination with living radical polymerization techniques. ${ }^{21,22,37}$ During such heterogeneous polymerizations, the reaction solution becomes distinctly turbid at the onset of micellar nucleation, since the growing colloidal particles scatter light. ${ }^{21-23,37}$ However, in the present work our initial experiments using a poly(methacrylic acid)based CTA to polymerize TFEMA did not lead to any significant increase in turbidity, which led us to believe that the PTFEMA block was actually soluble in ethanol at $70{ }^{\circ} \mathrm{C}$. Subsequent DLS and TEM experiments demonstrated that this interpretation was incorrect (see later). Moreover, in a control experiment, RAFT homopolymerization of TFEMA was conducted in ethanol at $70{ }^{\circ} \mathrm{C}$ using a small molecule RAFT CTA. This synthesis resulted in macroscopic precipitation at $70^{\circ} \mathrm{C}$, thus confirming beyond any doubt the insolubility of PTFEMA in ethanol at this temperature. Thus sterically-stabilized diblock copolymer nanoparticles are indeed formed when using an appropriate ethanol-soluble macro-CTA as originally anticipated, but little or no turbidity is observed due to the minimal contrast between the insoluble PTFEMA chains and ethanol, whose refractive indices are 1.41 and 1.36, respectively. In the present work, we show that this serendipitous finding provides an opportunity to compare the living character of RAFT polymerizations conducted under homogeneous solution and heterogeneous dispersion conditions. Various low polydispersity macro-CTAs (e.g. poly(methacrylic acid), PMAA or poly(2-(dimethylamino) ethyl methacrylate), PDMA) were synthesized by conventional RAFT solution polymerization. After isolation and purification, each macro-CTA was chain-extended in turn using TFEMA to produce amphiphilic diblock copolymers in the form of colloidally stable sterically-stabilized particles via RAFT dispersion polymerization in ethanol at $70{ }^{\circ} \mathrm{C}$ (see Scheme 1 and the Experimental section in the ESI $\dagger$ ). Initial attempts to use these macro-CTAs via RAFT dispersion polymerization of TFEMA in ethanol using ACVA initiator suffered from substantially

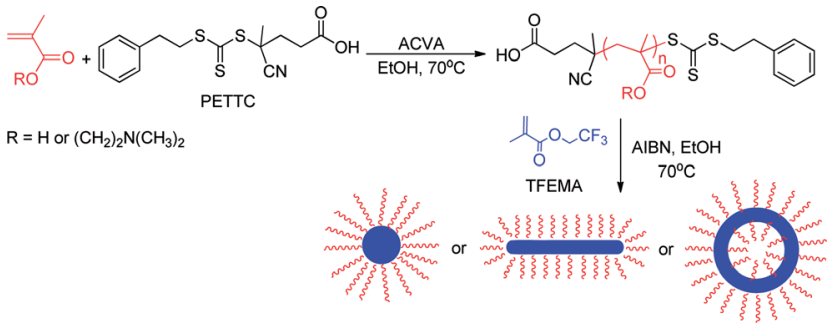

Scheme 1 RAFT dispersion polymerization of a relatively low refractive index monomer, 2,2,2-trifluoroethyl methacrylate (TFEMA), in ethanol at $20 \% \mathrm{w} / \mathrm{w}$ solids using either a poly(methacrylic acid) or a poly(2-(dimethylamino)ethyl methacrylate) macro-CTA at $70{ }^{\circ} \mathrm{C}$. The final diblock copolymer morphology can be spheres, worms or vesicles, depending on the precise block copolymer composition that is targeted.

incomplete monomer conversions (only $\sim 50-60 \%$ after $24 \mathrm{~h}$ at $70{ }^{\circ} \mathrm{C}$, as judged by ${ }^{1} \mathrm{H}$ NMR spectroscopy). Previously, we had postulated that the significant acceleration in the rate of polymerization of benzyl methacrylate observed after micellar nucleation was a result of partitioning of the monomer within the micelles to solubilize the growing chains. ${ }^{23}$ Bearing this particle growth mechanism in mind, the incomplete monomer conversions were considered to be most likely due to the insolubility of the anionic azo initiator in the semi-fluorinated TFEMA monomer, which would lead to exclusion of the former reagent from the micelles and hence cessation of the RAFT polymerization. This hypothesis proved to be correct: simply replacing the original anionic ACVA initiator with a neutral AIBN initiator led to much higher monomer conversions ( $>90 \%$ within $24 \mathrm{~h}$ at $70^{\circ} \mathrm{C}$ ). This optimized formulation for the RAFT dispersion polymerization of TFEMA in ethanol allows the production of well-defined block copolymer spheres, worms or vesicles with relatively low refractive indices, see Scheme 1.

Kinetic studies of the RAFT dispersion polymerization of TFEMA conducted in ethanol at $70{ }^{\circ} \mathrm{C}$ using a $\mathrm{PDMA}_{43}$ macroCTA indicated a final monomer conversion of $91 \%$ after $24 \mathrm{~h}$ using AIBN initiator (see Fig. 1). The almost perfect linearity over a wide range of monomer conversion indicates first-order kinetics. Similar kinetic data were obtained when using the PMAA $_{70}$ macro-CTA (see ESI, Fig. S1 $\dagger$ ). However, it is noteworthy that such high conversions were only achieved on this time scale by using a relatively low macro-CTA/initiator molar ratio of 2.50 in both cases. This is somewhat lower than the (macro-) CTA/initiator molar ratios of 5-10 that are normally utilized for RAFT solution polymerizations ${ }^{52-55}$ and is thus likely to lead to reduced control over the polymerization. ${ }^{56}$

At first sight, the evolution of number-average molecular weight $\left(M_{\mathrm{n}}\right)$ with conversion as determined by THF GPC [for PDMA-PTFEMA chains $v s$. poly(methyl methacrylate standards)] is not particularly linear. Moreover, the apparent polydispersity of the targeted PDMA $_{43}-$ PTFEMA $_{300}$ diblock copolymer at $91 \%$ conversion is around 1.76, (see Fig. 2). Taken at face value, these data suggest rather poor pseudo-living character. Indeed, inspecting the GPC curves obtained for the TFEMA polymerization indicated distinctly bimodal distributions (see Fig. 3). 


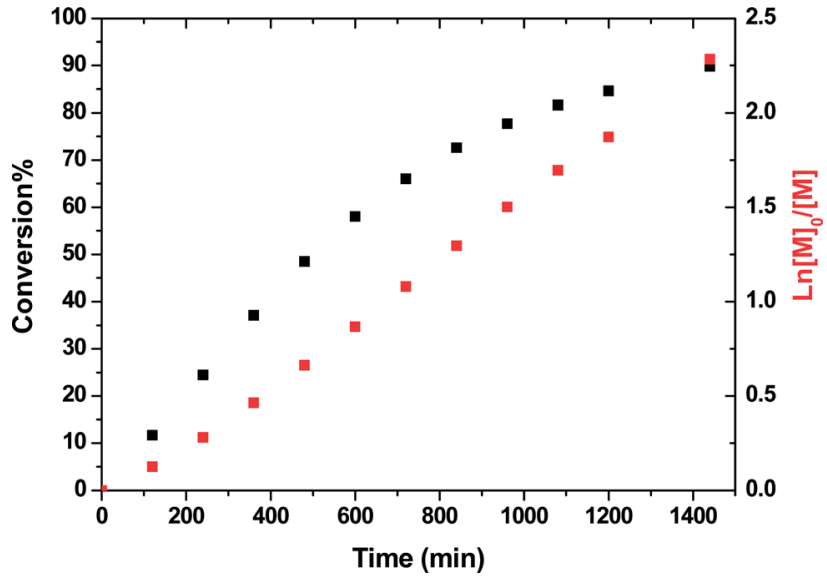

Fig. 1 Kinetic data obtained for the RAFT dispersion polymerization of TFEMA at $20 \% \mathrm{w} / \mathrm{w}$ solids in ethanol at $70{ }^{\circ} \mathrm{C}$ using a PDMA 43 macroCTA and a macro-CTA/AIBN molar ratio of 2.50. The targeted diblock composition was $\mathrm{PDMA}_{43}-\mathrm{PTFEMA}_{300 .}$

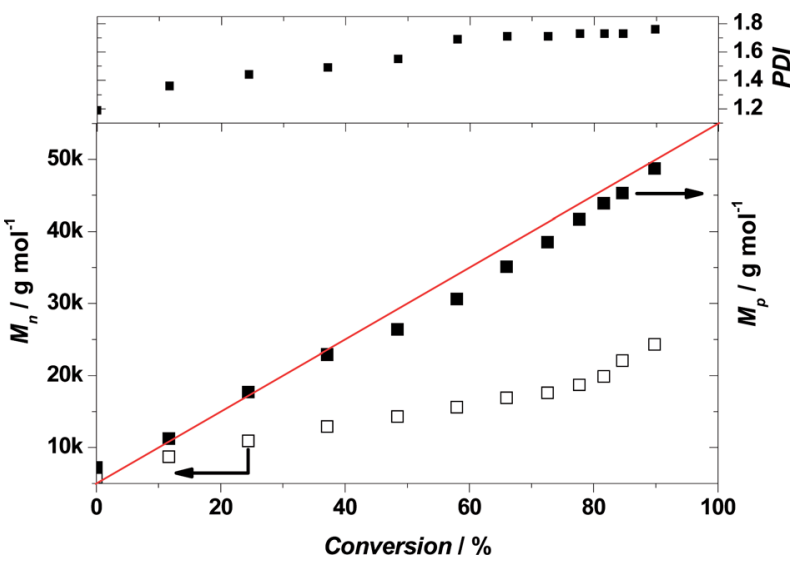

Fig. 2 Evolution of number-average molecular weight $\left(M_{n}\right)$, peak molecular weight $\left(M_{p}\right)$ and polydispersity $\left(M_{w} / M_{n}\right)$ with monomer conversion for the RAFT dispersion polymerization of TFEMA at 20\% $\mathrm{w} / \mathrm{w}$ solids in ethanol at $70{ }^{\circ} \mathrm{C}$ using a PDMA 43 macro-CTA and a macro-CTA/AIBN molar ratio of 2.50, as judged by THF GPC (vs. poly(methyl methacrylate) calibration standards). The targeted diblock composition was PDMA $43-$ PTFEMA $_{300 .}$.

However, a much more convincing linear plot can be obtained by plotting the peak molecular weight $\left(M_{\mathrm{p}}\right)$ for the major peak, rather than the $M_{\mathrm{n}}$ computed for the bimodal distribution (see Fig. 2). For the GPC analysis of conventional diblock copolymers using a refractive index detector, there is a tacit assumption that the two blocks have comparable refractive indices. Indeed, this is normally the case for most methacrylic comonomers. ${ }^{57}$ However, the refractive index of the PTFEMA block is 1.41; this is significantly lower than that of most non-fluorinated methacrylic polymers, which typically range from 1.49 to $1.59 .{ }^{57}$ This leads to the refractive index detector significantly underestimating the signal intensity due to the semi-fluorinated block relative to that of the PDMA (or PMAA). This in turn exaggerates the apparent contamination of the diblock copolymer by the macro-CTA, as exemplified in Fig. 3. Similar GPC observations

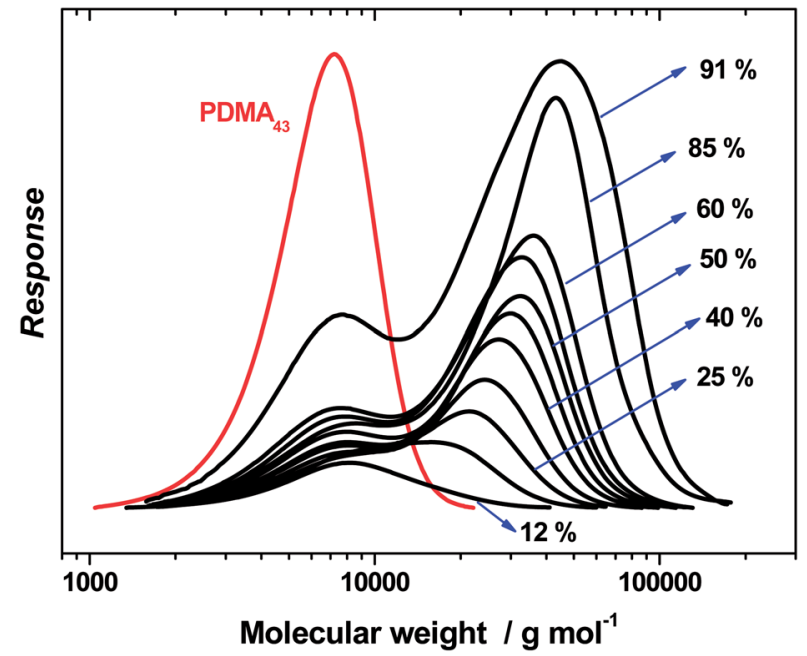

Fig. 3 GPC curves (refractive index detector) recorded during the RAFT dispersion polymerization of TFEMA at $20 \% \mathrm{w} / \mathrm{w}$ solids in ethanol at $70{ }^{\circ} \mathrm{C}$ using a PDMA 43 macro-CTA and a macro-CTA/AIBN molar ratio of 2.50. The targeted diblock composition was $\mathrm{PDMA}_{43}-$ PTFEMA $_{300}$.

were also made during the synthesis of the $\mathrm{PMAA}_{70}-\mathrm{PTFEMA}_{300}$ diblock copolymer (see Fig. S2-S4 in the ESI $\dagger$ ). In this latter case, exhaustive methylation of the PMAA block was required prior to GPC analysis.

In order to address this problem, the final bimodal GPC curve shown in Fig. 3 was deconvoluted to give two unimodal components comprising the $\mathrm{PDMA}_{43}$ macro-CTA (red curve) and the PDMA $_{43}-$ PTFEMA $_{273}$ diblock copolymer obtained at $91 \%$ conversion (black curve), see Fig. 4a. These delineated GPC signals were then corrected for their respective refractive indices (see Fig. $4 \mathrm{~b}$ ) and recombined to produce a more physically realistic GPC curve (see inset in Fig. 4b), which now possesses minimal bimodality. The polydispersity of this corrected GPC curve was calculated to be approximately 1.31 , which is comparable to that observed for the RAFT alcoholic dispersion polymerization of benzyl methacrylate..$^{26,28}$ Thus these recalculated GPC data indicate that relatively good pseudo-living character is obtained for the RAFT dispersion polymerization of TFEMA in ethanol under the stated reaction conditions.

Both McCormick's group ${ }^{58,59}$ and Laschewsky and coworkers ${ }^{53,60}$ have reported that RAFT solution polymerizations conducted in aqueous media using dithiobenzoate-based RAFT CTAs can be prone to in situ hydrolysis under certain conditions, which leads to the progressive loss of chain-end fidelity. Similar side-reactions occur for RAFT polymerizations conducted in other solvents under monomer-starved conditions. In the present work, a trithiocarbonate-based CTA was utilized, which gives rise to a distinctive absorption band at $305 \mathrm{~nm} .{ }^{61} \mathrm{In}$ principle, monitoring the intensity of this spectral feature over time should allow the gradual loss of trithiocarbonate endgroups during a RAFT solution polymerization to be assessed. However, this approach is not normally applicable for RAFT polymerizations conducted under heterogeneous conditions. This is because the scattered light intensity from the growing 

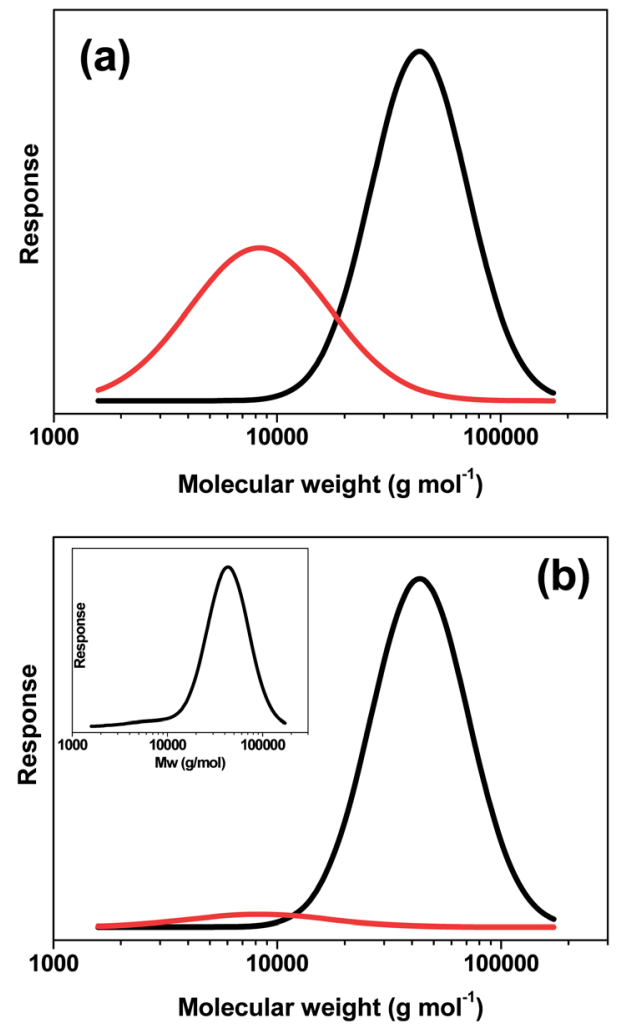

Fig. 4 (a) Deconvoluted refractive index GPC curves obtained for the last kinetic sample (at 91\% conversion, see Fig. 3) obtained when targeting a PDMA $43-P T F E M A_{300}$ diblock copolymer (the red curve represents the PDMA 43 macro-CTA); (b) the same deconvoluted GPC curves corrected for the relatively large refractive index difference between the PDMA 43 macro-CTA $(R I=1.54)$ and the PTFEMA block $(R I=1.41)$. The inset shows the recombined refractive index-corrected GPC traces.

colloidal nanoparticles is proportional to $1 / \lambda^{4}$, which produces a rising baseline at lower wavelengths that precludes accurate quantification. Fortuitously, the refractive index of 1.41 for the semi-fluorinated PTFEMA block ${ }^{57}$ is sufficiently close to that for the ethanol solvent $(1.36)^{57}$ so as to minimize this light scattering problem. It is also feasible that, for these stericallystabilized nanoparticles, the PDMA (or PMAA) stabilizer chains may increase the effective refractive index of the ethanol, thus further reducing the refractive index difference between the nanoparticles and the solvent and hence reducing the light scattering. In any case it is clear that, provided that the final diblock copolymer nanoparticle dimensions are not too large, the PTFEMA/ethanol combination utilized in the present study offers a rare opportunity to compare the living character of RAFT dispersion polymerization at $20 \% \mathrm{w} / \mathrm{w}$ solids with that of RAFT solution polymerization performed under the same conditions using UV-visible absorption spectroscopy.

It is well known that the dimensions of diblock copolymer nano-objects prepared via PISA formulations are sensitive to the mean degrees of polymerization (DP) of each block..$^{22,37}$ If the stabilizer block DP is relatively long, then only relatively small, near-monodisperse spherical nanoparticles are produced. ${ }^{26,28}$ However, if the stabilizer block DP is relatively short, then targeting a high DP for the core-forming block can produce either worms or vesicles. ${ }^{23,24,26,28}$ The colloidal dimensions of these latter nano-objects are sufficiently large to efficiently scatter visible light, resulting in rather turbid dispersions, even for relatively small differences in refractive index between the solvent and the copolymer. Thus, in the present work relatively high stabilizer DPs were targeted to ensure that only spherical diblock copolymer nanoparticles with mean diameters well below $100 \mathrm{~nm}$ were obtained when performing the UV-visible absorption spectroscopy studies.

This strategy proved to be effective: TEM studies indicated that well-defined spherical nanoparticles were obtained when using either the $\mathrm{PMAA}_{70}$ or the PDMA 94 macro-CTA (see Fig. 5a and $b$ ). This was confirmed by DLS studies, which indicated final intensity-average particle diameters of around $70 \mathrm{~nm}$ $(\mathrm{PDI}=0.041)$ and $50 \mathrm{~nm}(\mathrm{PDI}=0.032)$ for the $\mathrm{PMAA}_{70^{-}}$ PTFEMA $_{273}$ and PDMA $_{94}-$ PTFEMA $_{282}$ nanoparticles, respectively. In contrast, using a shorter PDMA ${ }_{43}$ macro-CTA led to the in situ evolution from spheres to worms to polydisperse vesicles (see Fig. 5c) during the TFEMA polymerization because of the gradual reduction in the molecular curvature of the growing copolymer chains. ${ }^{23}$ Such changes in diblock copolymer morphology lead to a pronounced increase in the turbidity of the reaction mixture, which precludes quantitative UV-visible spectroscopy studies (see Fig. S7†).

A linear calibration curve was constructed for the trithiocarbonate-based PETTC RAFT agent, see Fig. 6. The molar extinction coefficient, $\varepsilon$, at $305 \mathrm{~nm}$ for this compound was calculated to be $15064 \pm 8 \mathrm{~L} \mathrm{~mol}^{-1} \mathrm{~cm}^{-1}$, which is close to the literature value of $14500 \mathrm{~L} \mathrm{~mol}^{-1} \mathrm{~cm}^{-1}$ reported by Laschewsky and co-workers for a similar trithiocarbonate species. ${ }^{61} \mathrm{~A}$ final diblock composition of $\mathrm{PMAA}_{70}-\mathrm{PTFEMA}_{300}$ was targeted under otherwise identical conditions $\left(20 \% \mathrm{w} / \mathrm{w}\right.$ solids, $70{ }^{\circ} \mathrm{C}$, macroCTA/AIBN molar ratio $=2.50$ ) via either RAFT dispersion polymerization in ethanol or RAFT solution polymerization in THF and UV-visible absorption spectroscopy was used to assess chain-end fidelity in each case. In both cases, progressive attenuation of the $305 \mathrm{~nm}$ band was observed during the RAFT polymerization (see Fig. 7 ). However, around $27 \%$ of the RAFT chain-ends were lost within $24 \mathrm{~h}$ during dispersion polymerization, whereas approximately $43 \%$ of the original chain-ends were destroyed during the equivalent solution polymerization (see Fig. 8a). This relatively high loss of chain-end fidelity is due in part to the relatively low macro-CTA/initiator molar ratio

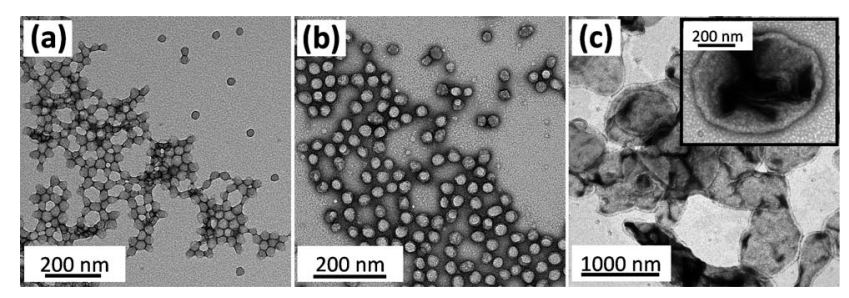

Fig. 5 TEM images for various diblock copolymer nanoparticles prepared by RAFT dispersion polymerization of TFEMA at $20 \% \mathrm{w} / \mathrm{w}$ solids in ethanol at $70{ }^{\circ} \mathrm{C}$ : (a) $\mathrm{PMAA}_{70}-\mathrm{PTFEMA}_{273}$; (b) $\mathrm{PDMA}_{94}-$ PTFEMA $_{282}$; (c) PDMA $43-$ PTFEMA $_{273}$. 


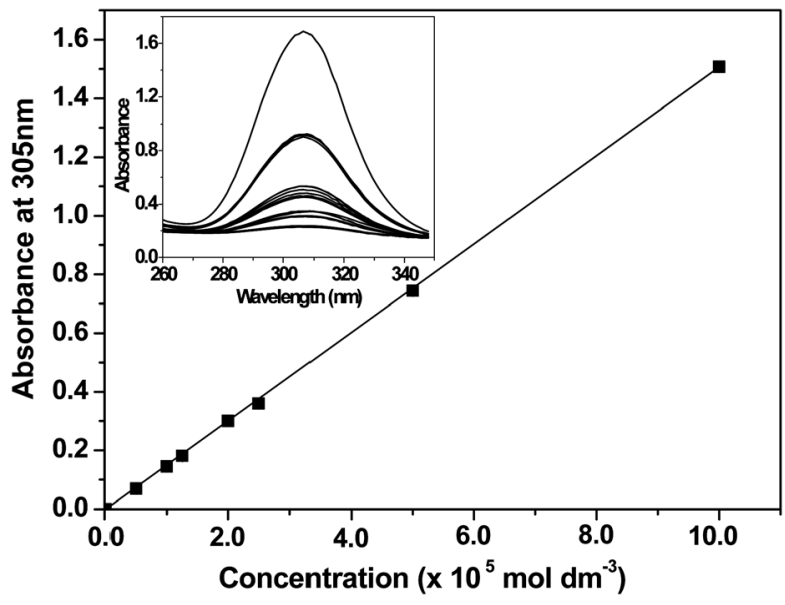

Fig. 6 Beer-Lambert calibration curve for S-phenylethyl- $S^{\prime}-\left(\alpha, \alpha^{\prime}-\right.$ dimethyl- $\alpha^{\prime \prime}$-acetic acid)trithiocarbonate $\left(\varepsilon=15064 \pm 8 \mathrm{~L} \mathrm{~mol}^{-1}\right.$ $\mathrm{cm}^{-1}$ ) obtained using UV-visible absorption spectroscopy at a $\lambda_{\max }$ of $305 \mathrm{~nm}$
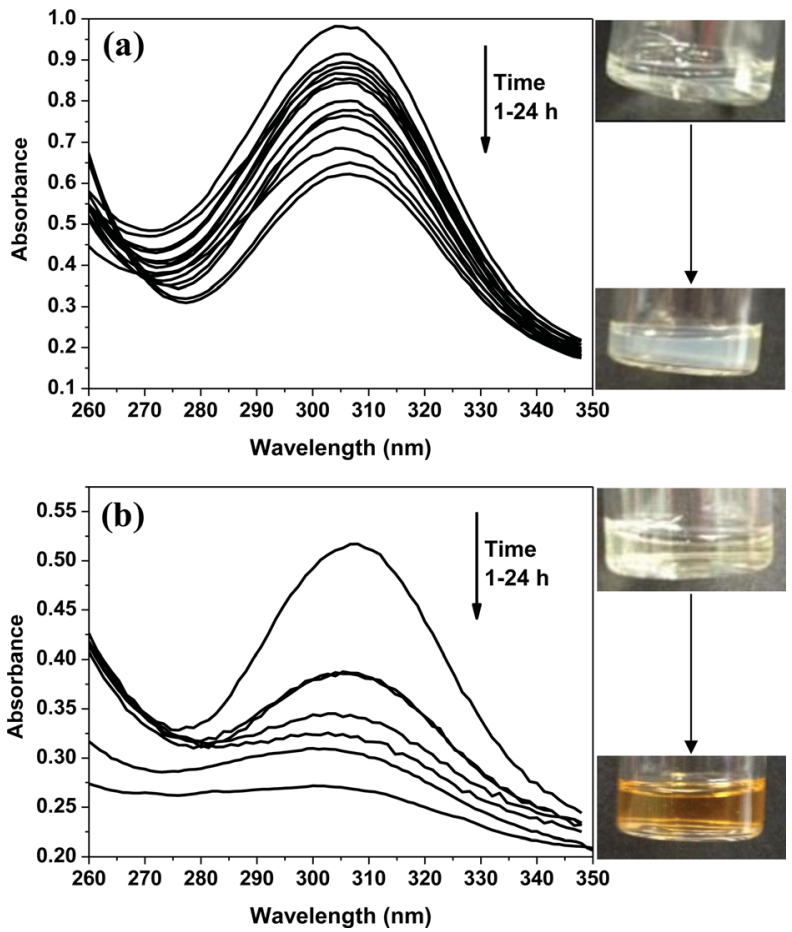

Fig. 7 (a) UV-visible absorption spectra recorded during the RAFT dispersion polymerization of TFEMA at $20 \% \mathrm{w} / \mathrm{w}$ solids in ethanol at 70 ${ }^{\circ} \mathrm{C}$ when targeting a final diblock composition of PMAA $\mathrm{P}_{70}-\mathrm{PTFEMA}_{300}$. The inset photo shows the relatively low turbidity observed for the final kinetic sample obtained at $91 \%$ conversion. (b) UV-visible absorption spectra recorded during the RAFT solution polymerization of TFEMA at $20 \% \mathrm{w} / \mathrm{w}$ solids in THF at $70{ }^{\circ} \mathrm{C}$ using a $\mathrm{PMAA}_{70}$ macro-CTA when

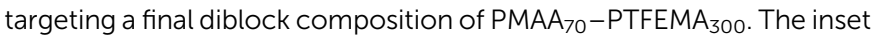
photo shows the transparent orange reaction solution observed for the final kinetic sample obtained at $91 \%$ conversion.

used in these syntheses, which was chosen to ensure relatively high monomer conversions within reasonable reaction times (i.e. within $24 \mathrm{~h}$ ).
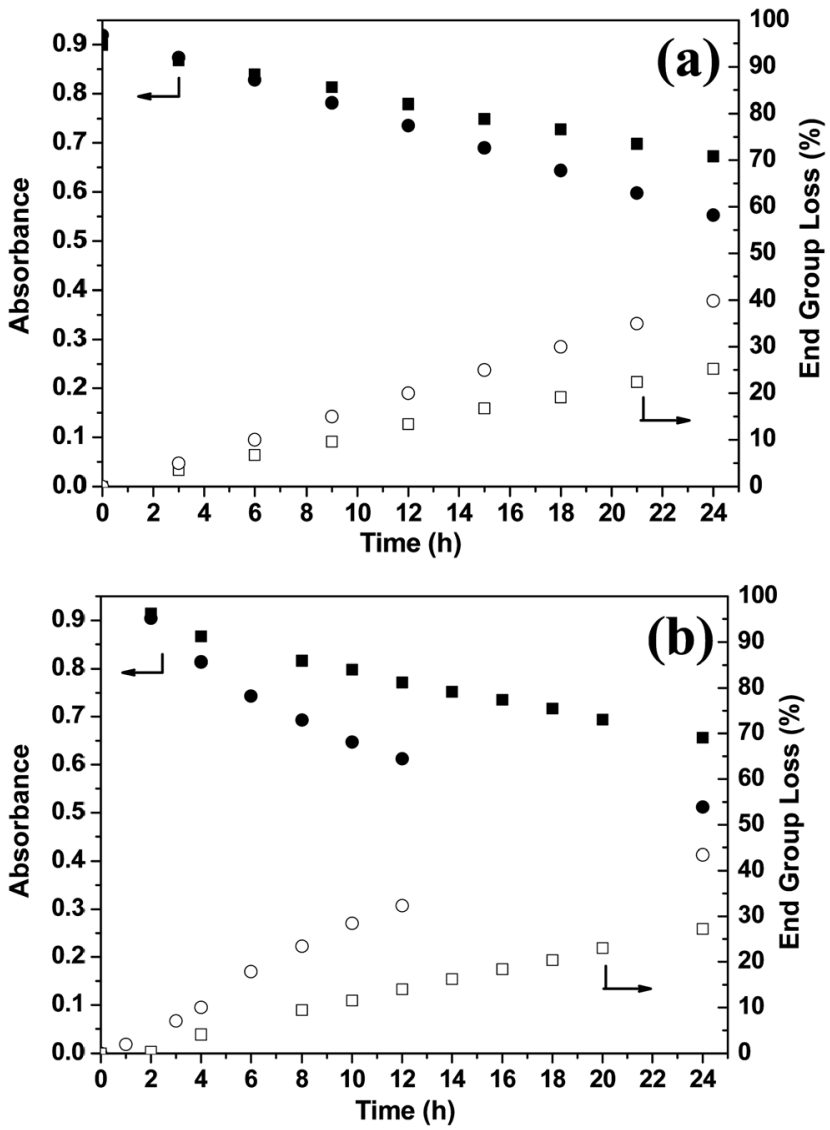

Fig. 8 Comparison of the RAFT chain-end fidelity observed during the RAFT dispersion polymerization of TFEMA in ethanol and the corresponding RAFT solution polymerization of TFEMA in THF using UV-visible absorption spectroscopy. The observed absorbance (at 305 $\mathrm{nm}$ ) is indicated by the filled symbols, while the corresponding calculated loss of RAFT chain-ends is given by the open symbols. Both RAFT syntheses were conducted at $70^{\circ} \mathrm{C}$, the total solids content was $20 \% \mathrm{w} / \mathrm{w}$ and the targeted diblock composition was (a) $\mathrm{PDMA}_{94}-$ PTFEMA $_{300}$ and (b) $\mathrm{PMAA}_{70}-\mathrm{PTFEMA}_{300}$.

Remarkably similar UV-visible spectroscopic data were obtained when targeting a diblock composition of $\mathrm{PDMA}_{94^{-}}$ PTFEMA $_{300}$ via RAFT dispersion or RAFT solution polymerization under the same conditions (see Fig. $\mathrm{S} 5 \dagger$ and $8 \mathrm{~b}$ ). For both formulations, the final monomer conversion was at least $94 \%$ and (uncorrected) THF GPC analysis indicated almost identical $M_{\mathrm{n}}$ and $M_{\mathrm{w}} / M_{\mathrm{n}}$ values (see Table S1 $\dagger$ ). This suggests that the striking difference in chain-end fidelity observed for the homogeneous and heterogeneous reaction conditions is genuine and also indicates that it is independent of the nature of the macro-CTA stabilizer. ${ }^{62}$ Given that the distinct change in color (from pale yellow to orange-brown) observed during the RAFT solution polymerization is not seen for the RAFT dispersion polymerization, it is tempting to suggest that the latter formulation somehow suppresses one or more degradation pathways. However, the mechanism(s) of chemical degradation in RAFT polymerizations are believed to be complex, so such speculation may not be justified..$^{53,58}$ An alternative explanation may be that the relatively high local concentration of TFEMA 
monomer within the growing diblock copolymer micelles simply delays the onset of monomer-starved conditions, which otherwise leads to a loss of active chain-ends. ${ }^{63,64}$

It is also noteworthy that there have been several recent reports suggesting that enhanced control can be achieved over radical polymerizations conducted under heterogeneous conditions. ${ }^{\mathbf{6 5 6}}$ Nevertheless, it is clear that a RAFT dispersion polymerization can offer significantly greater living character than the equivalent RAFT solution polymerization, which is likely to be advantageous for, say, the synthesis of ABC triblock copolymers. $^{25}$

Spherical block copolymer nanoparticles were also prepared using the anionic $\mathrm{PMAA}_{70}$ and cationic $\mathrm{PDMA}_{43}$ macro-CTAs by targeting somewhat shorter DPs for the core-forming PTFEMA block. In each case, these nanoparticles were transferred from ethanol into water via equilibrium dialysis without any loss of colloidal stability and subsequently characterized by aqueous electrophoresis (see Fig. 9). As expected, the PMAA $_{70}-$ PTFEMA $_{80}$ nanoparticles ( $42 \mathrm{~nm}$ diameter by DLS) exhibited negative zeta potentials (around $-30 \mathrm{mV}$ ) over the entire $\mathrm{pH}$ range from 6 to 10 , with some evidence for an isoelectric point at approximately $\mathrm{pH} 2$. In contrast, $\mathrm{PDMA}_{43}-\mathrm{PTFEMA}_{80}$ nanoparticles $(35 \mathrm{~nm}$ diameter by DLS) exhibited positive zeta potentials of around $+40 \mathrm{mV}$ below neutral $\mathrm{pH}$, with an isoelectric point being observed at around $\mathrm{pH} 9$.

In both cases, the electrophoretic footprint of the nanoparticles is dictated by the weak polyacid (or polybase) nature of the steric stabilizer chains. Very similar electrophoretic behavior has been reported previously for related block copolymer nanoparticles synthesized when using PMAA and PDMA macro-CTAs for the RAFT dispersion polymerization of benzyl methacrylate in alcoholic media. ${ }^{26,28}$ Thus RAFT dispersion polymerization is a convenient formulation for the preparation of well-defined semi-fluorinated spherical nanoparticles with either highly cationic or anionic character.

Previously, it has been suggested by both ourselves ${ }^{23,24}$ and others $^{34}$ that the growing block copolymer nanoparticles

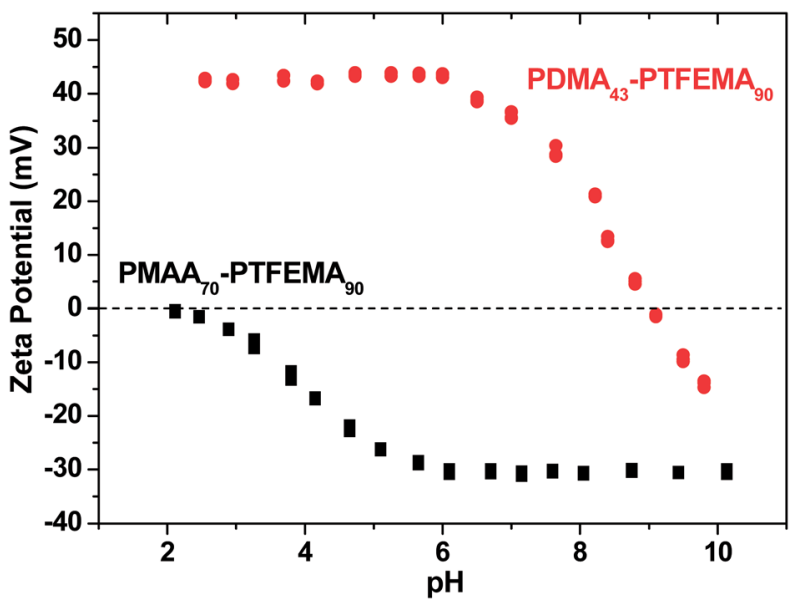

Fig. 9 Aqueous electrophoresis curves obtained for the following spherical diblock copolymer nanoparticles: PMAA ${ }_{70}-$ PTFEMA $_{80}(\mathbf{\square})$ and $\mathrm{PDMA}_{43}-\mathrm{PTFEMA}_{80}$ (๑). Zeta potential measurements were performed on $0.01 \mathrm{wt} \%$ aqueous dispersions in the presence of $10^{-3} \mathrm{M} \mathrm{NaCl}$. produced during RAFT dispersion polymerization most likely become monomer-swollen after micellar nucleation has occurred. If this is correct, then the higher local monomer concentration would account for the pronounced rate enhancement that usually coincides with the onset of particle formation. Moreover, the unreacted monomer within the swollen micelles ensures that the propagating copolymer chains have sufficient mobility to enable the in situ evolution of the copolymer morphology from spheres to worms to vesicles, as is often observed for such formulations. ${ }^{23,24}$ In principle, this 'monomer-swollen micelle' hypothesis could be examined by ${ }^{1} \mathrm{H}$ NMR spectroscopy studies for RAFT dispersion polymerizations conducted in deuterated solvents. In practice, we are unaware of any such studies. However, the semi-fluorinated TFEMA monomer offers an interesting opportunity to conduct similar, but rather more convenient, experiments utilizing ${ }^{19} \mathrm{~F}$ NMR spectroscopy in conventional protonated solvents. Moreover, the ${ }^{19} \mathrm{~F}$ nucleus is $100 \%$ abundant, which offers excellent sensitivity compared to ${ }^{13} \mathrm{C}$ NMR spectroscopy. A series of stacked ${ }^{19} \mathrm{~F}$ NMR spectra are shown in Fig. 10. TFEMA monomer alone produces an intense triplet signal with two side bands at around $-75 \mathrm{ppm}$. Like most vinyl monomers, TFEMA is a good solvent for its corresponding homopolymer. As expected, the PTFEMA homopolymer signal is somewhat broader and shifted to around -74 ppm; hence it is readily distinguishable from that due to TFEMA monomer. A similar homopolymer signal can be observed on addition of TFEMA monomer to a PMAA $_{70}-$ PTFEMA $_{300}$ diblock copolymer, which forms PTFEMA-core micelles in ethanol. A comparable homopolymer signal is also obtained for a kinetic sample taken at around $50 \%$ conversion when targeting a final diblock composition of $\mathrm{PMAA}_{70}-\mathrm{PTFEMA}_{300}$. At 91\% conversion, this homopolymer signal is significantly attenuated since there is no longer sufficient TFEMA monomer available to ensure full solvation of the PTFEMA chains. In summary, these ${ }^{19} \mathrm{~F}$ NMR spectroscopy studies provide the first direct experimental evidence for the formation of monomer-swollen block copolymer micelles during RAFT dispersion polymerization at $70{ }^{\circ} \mathrm{C}$.

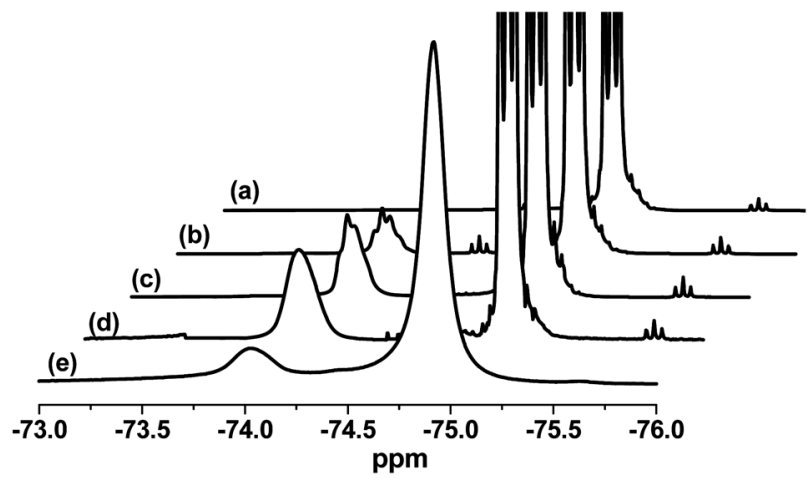

Fig. $10{ }^{19} \mathrm{~F}$ NMR spectra recorded at $20{ }^{\circ} \mathrm{C}$ in ethanol (in the presence of a small amount of $\mathrm{CD}_{3} \mathrm{OD}$ ) for: (a) TFEMA monomer; (b) PTFEMA ${ }_{53}+$ TFEMA monomer; (c) PMAA $70-$ PTFEMA 300 + TFEMA monomer; (d) $\mathrm{PMAA}_{70}-\mathrm{PTFEMA}_{150}$ (i.e. a kinetic sample taken at $50 \%$ monomer conversion when targeting $\mathrm{PMAA}_{70}-\mathrm{PTFEMA}_{300}$ ); (e) $\mathrm{PMAA}_{70}-$ $\mathrm{PTFEMA}_{273}$ (i.e. a kinetic sample taken at $91 \%$ monomer conversion when targeting $\mathrm{PMAA}_{70}-\mathrm{PTFEMA}_{300}$ ). 


\section{Conclusions}

We report the first example of the RAFT polymerization of a semi-fluorinated monomer, 2,2,2-trifluoroethyl methacrylate, using an alcoholic dispersion polymerization formulation. The fortuitous refractive index match between this low refractive index monomer and the ethanol solvent leads to the formation of block copolymer nanoparticles with minimal turbidity owing to the very weak light scattering. This provides a rare opportunity to compare the living character of RAFT polymerizations conducted under homogeneous solution and heterogeneous dispersion conditions. Using UV-visible absorption spectroscopy to monitor the loss of RAFT CTA chain-ends during the course of the polymerization, we find that a significantly higher proportion of the original trithiocarbonate groups (at least 73$75 \%$ ) survive under dispersion polymerization conditions, compared to only $55-60 \%$ for the corresponding RAFT solution polymerization conducted in THF. This is consistent with the RAFT dispersion polymerization data recently reported by ourselves and others..$^{23,25,27,29,30,34}$ Moreover, the semi-fluorinated nature of the TFEMA also enables convenient ${ }^{19}$ F NMR experiments to be conducted; this spectroscopic technique provides direct evidence for our earlier hypothesis that RAFT dispersion polymerization proceeds via monomer-swollen block copolymer micelles. These observations are consistent with the pronounced rate accelerations previously reported for related RAFT dispersion polymerization formulations. ${ }^{23,24,26,28-30,34,44,46}$

\section{Experimental}

\section{Materials}

All reagents were purchased from Sigma-Aldrich (UK) and used as received unless otherwise noted. THF (>99.8\%) and absolute ethanol (99.8\%) were supplied by Fisher and VWR, respectively. 4,4'-Azobis-4-cyanopentanoic acid (ACVA) and 4,4'-azobis-(2methylpropionitrile) (AIBN) were used as initiators. 4-Cyano-4(2-phenylethane sulfanylthiocarbonyl)sulfanylpentanoic acid (PETTC) was synthesized as reported previously. ${ }^{29}$

\section{Copolymer characterization}

Molar mass and molar mass distributions of the block copolymers were measured using size exclusion chromatography (SEC); the set-up comprised two $5 \mu \mathrm{m}(30 \mathrm{~cm})$ mixed C columns, a WellChrom K-2301 refractive index detector operating at 950 $\pm 30 \mathrm{~nm}$, and the eluent was THF [containing $2 \mathrm{v} / \mathrm{v} \%$ triethylamine and $0.05 \mathrm{w} / \mathrm{v} \%$ butylhydroxytoluene (BHT)] at a flow rate of $1.0 \mathrm{~mL} \mathrm{~min}^{-1}$; a series of ten near-monodisperse linear poly(methyl methacrylates) $\left(M_{\mathrm{p}}\right.$ ranging from 1280 to $330000 \mathrm{~g}$ $\mathrm{mol}^{-1}$ ) were purchased from Polymer Labs (UK) and employed as calibration standards with the above refractive index detector. ${ }^{1} \mathrm{H}$ NMR spectra were acquired on a Bruker $250 \mathrm{MHz}$ or $400 \mathrm{MHz}$ in $\mathrm{CDCl}_{3} \cdot{ }^{19} \mathrm{~F}$ NMR spectra were acquired on a Bruker $250 \mathrm{MHz}$ spectrometer in ethanol (in the presence of a small amount of $\mathrm{CD}_{3} \mathrm{OD}$ to provide a signal lock). All chemical shifts are reported in ppm $(\delta)$. TEM studies were conducted using a Philips CM 100 instrument operating at $100 \mathrm{kV}$. To prepare TEM samples, $5.0 \mu \mathrm{L}$ of a dilute aqueous copolymer solution was placed onto a carbon-coated copper grid, stained with uranyl formate and dried under ambient conditions. DLS measurements were conducted using a Malvern Instruments Zetasizer Nano series instrument equipped with a $4 \mathrm{~mW}$ He-Ne laser operating at $633 \mathrm{~nm}$, an avalanche photodiode detector with high quantum efficiency, and an ALV/LSE-5003 multiple tau digital correlator electronics system. Aqueous electrophoresis measurements were performed on a 0.01 wt $\%$ aqueous copolymer solution using the same Malvern Instruments Zetasizer Nano series instrument. The solution $\mathrm{pH}$ was adjusted by the addition of $0.01 \mathrm{M} \mathrm{HCl}$ or $0.01 \mathrm{M} \mathrm{KOH}$ using an autotitrator. The UV-visible spectra were recorded with a $1.0 \mathrm{~cm}$ quartz cuvette using a Cary 3 Bio spectrometer operating at a scan speed of $600 \mathrm{~nm} \mathrm{~min}^{-1}$.

\section{Synthesis of poly(methacrylic acid) (PMAA) macro-CTA}

In a typical experiment, a round-bottomed flask was charged with MAA (5.00 g; $58.0 \mathrm{mmol}$ ), PETTC (300 mg; $0.890 \mathrm{mmol}$ ), ACVA $(50.0 \mathrm{mg}, 0.179 \mathrm{mmol})$ and ethanol $(5.00 \mathrm{~g})$. The sealed reaction vessel was purged with nitrogen and placed in a preheated oil bath at $70{ }^{\circ} \mathrm{C}$ for $3 \mathrm{~h}$. The resulting PMAA macro-CTA (MAA conversion $=100 \%$; after exhaustive methylation, $M_{\mathrm{n}}=$ $\left.9000 \mathrm{~g} \mathrm{~mol}^{-1}, M_{\mathrm{w}}=11000 \mathrm{~g} \mathrm{~mol}^{-1}, M_{\mathrm{w}} / M_{\mathrm{n}}=1.19\right)$ was purified using dialysis, first against a $1: 1$ water : methanol mixture and then against deionized water. The polymer was isolated by freeze-drying from this aqueous solution overnight. A mean DP of 70 was calculated for this macro-CTA using ${ }^{1} \mathrm{H}$ NMR spectroscopy by comparing the integrated signal intensity due to the aromatic protons at 7.2-7.4 ppm with that due to the methacrylic polymer backbone at $0.4-2.5 \mathrm{ppm}$.

\section{Synthesis of poly(2-(dimethylamino)ethyl methacrylate) (PDMA) macro-CTA}

A round-bottomed flask was charged with 2-(dimethylamino) ethyl methacrylate (DMA; $10.0 \mathrm{~g}, 32 \mathrm{mmol}$ ), PETTC (0.216 g, 0.64 mmol), ACVA (36 mg, $0.127 \mathrm{mmol}$ ), and THF (10.0 g) (target $\mathrm{DP}=50$ ). The sealed reaction vessel was purged with nitrogen and placed in a preheated oil bath at $70{ }^{\circ} \mathrm{C}$ for $6 \mathrm{~h}$. The resulting polymer (monomer conversion $=80 \% ; M_{\mathrm{n}}=6000 \mathrm{~g} \mathrm{~mol}^{-1}$, $M_{\mathrm{w}} / M_{\mathrm{n}}=1.22$ ) was purified by precipitation into excess petroleum ether. The mean degree of polymerization (DP) of this PDMA macro-CTA was calculated to be 43 using ${ }^{1} \mathrm{H}$ NMR spectroscopy by comparing the integrated signals corresponding to the aromatic protons at 7.2-7.4 ppm with those due to the methacrylic polymer backbone at 0.4-2.5 ppm.

\section{Synthesis of poly(methacrylic acid)-poly(2,2,2-trifluoroethyl methacrylate) (PMAA-PTFEMA) diblock copolymer particles via RAFT dispersion polymerization in ethanol at $70{ }^{\circ} \mathrm{C}$}

In a typical $20 \% \mathrm{w} / \mathrm{w}$ alcoholic dispersion polymerization synthesis of PMAA $_{70}-$ PTFEMA $_{300}$, TFEMA (1.50 g; $8.90 \mathrm{mmol}$ ), AIBN (2.00 mg; $0.012 \mathrm{mmol}$ ) and PMAA $_{70}$ macro-CTA (0.179 g; $0.030 \mathrm{mmol}$ ) were dissolved in ethanol $(6.72 \mathrm{~g})$. The reaction mixture was sealed in a round-bottomed flask, purged with 
nitrogen for $15 \mathrm{~min}$ and then placed in a pre-heated oil bath at $70{ }^{\circ} \mathrm{C}$ for $24 \mathrm{~h}$.

Synthesis of poly(2-(dimethylamino)ethyl methacrylate)poly(2,2,2-trifluoroethyl methacrylate) (PDMA-PTFEMA) diblock copolymer particles via RAFT dispersion polymerization in ethanol at $70{ }^{\circ} \mathrm{C}$

In a typical $20 \% \mathrm{w} / \mathrm{w}$ alcoholic dispersion polymerization synthesis of PDMA $_{43}-$ PTFEMA $_{300}$, TFEMA (1.50 g; $8.90 \mathrm{mmol}$ ), AIBN (2.00 mg; $0.012 \mathrm{mmol}$ ) and PDMA $_{43}$ macro-CTA (0.200 g; $0.030 \mathrm{mmol})$ were dissolved in ethanol $(6.81 \mathrm{~g})$. The reaction mixture was sealed in a round-bottomed flask, purged with nitrogen for $15 \mathrm{~min}$ and then placed in a pre-heated oil bath at $70{ }^{\circ} \mathrm{C}$ for $24 \mathrm{~h}$. Kinetic studies were conducted as follows. TFEMA (2.00 g; $11.9 \mathrm{mmol}$ ), AIBN (2.60 mg; $0.015 \mathrm{mmol}$ ) and PDMA $_{94}$ macro-CTA $(0.586 \mathrm{~g} ; 0.039 \mathrm{mmol})$ were co-dissolved in ethanol (10.35 g). This reaction mixture was sealed in a roundbottomed flask, purged with nitrogen for $30 \mathrm{~min}$, and then placed in a pre-heated oil bath at $70{ }^{\circ} \mathrm{C}$. Aliquots $(0.40 \mathrm{~mL})$ were extracted on an hourly basis under a positive nitrogen atmosphere for $24 \mathrm{~h}$. UV-visible spectra were recorded by diluting 20 $\mu \mathrm{L}$ of an aliquot in $2.0 \mathrm{~mL}$ ethanol. Each reading was repeated three times and the average value was reported.

Synthesis of poly(2-(dimethylamino)ethyl methacrylate)poly(2,2,2-trifluoroethyl methacrylate) (PDMA-PTFEMA) diblock copolymer particles via RAFT dispersion polymerization in ethanol at $70{ }^{\circ} \mathrm{C}$

In a typical $20 \% \mathrm{w} / \mathrm{w}$ alcoholic dispersion polymerization synthesis of PDMA ${ }_{94}-$ PTFEMA $_{300}$, TFEMA (1.50 g; $8.90 \mathrm{mmol}$ ), AIBN (2.00 mg; $0.012 \mathrm{mmol}$ ) and $\mathrm{PDMA}_{94}$ macro-CTA (0.440 g; $0.030 \mathrm{mmol}$ ) were dissolved in ethanol $(7.77 \mathrm{~g})$. The reaction mixture was sealed in a round-bottomed flask, purged with nitrogen for $15 \mathrm{~min}$ and then placed in a pre-heated oil bath at $70{ }^{\circ} \mathrm{C}$ for $24 \mathrm{~h}$.

Synthesis of poly(methacrylic acid)-poly(2,2,2-trifluoroethyl methacrylate) (PMAA-PTFEMA) diblock copolymer particles via RAFT solution polymerization in THF at $70{ }^{\circ} \mathrm{C}$

In a typical $20 \% \mathrm{w} / \mathrm{w}$ solution polymerization of $\mathrm{PMAA}_{70^{-}}$ PTFEMA $_{300}$, TFEMA (1.00 g; $5.90 \mathrm{mmol}$ ), AIBN (1.30 mg; 0.008 mmol) and PMAA $_{70}$ macro-CTA $(0.119 \mathrm{~g} ; 0.020 \mathrm{mmol})$ were dissolved in THF (4.48 g). The reaction mixture was sealed in a round-bottomed flask, purged with nitrogen for $15 \mathrm{~min}$ and then placed in a pre-heated oil bath at $70{ }^{\circ} \mathrm{C}$ for $14 \mathrm{~h}$. At this point, a further charge of AIBN (1.30 mg; $0.008 \mathrm{mmol}$ ) dissolved in ethanol $(0.10 \mathrm{~mL})$ was added as a fresh radical source. Reactions were quenched after $24 \mathrm{~h}$, at which point high monomer conversions were obtained.

Synthesis of poly(2-(dimethylamino)ethyl methacrylate)poly(2,2,2-trifluoroethyl methacrylate) (PDMA-PTFEMA) diblock copolymer particles via RAFT solution polymerization in THF at $70^{\circ} \mathrm{C}$

In a typical $20 \% \mathrm{w} / \mathrm{w}$ solution polymerization synthesis of PDMA $_{94}-$ PTFEMA $_{300}$, TFEMA (1.00 g; $\left.4.95 \mathrm{mmol}\right)$, AIBN (1.30 $\mathrm{mg} ; 0.008 \mathrm{mmol}$ ) and PDMA $_{94}$ macro-CTA (0.293 g; $\left.0.020 \mathrm{mmol}\right)$ were dissolved in ethanol $(5.18 \mathrm{~g})$. The reaction mixture was sealed in a round-bottomed flask, purged with nitrogen for 15 min and then placed in a pre-heated oil bath at $70{ }^{\circ} \mathrm{C}$ for $24 \mathrm{~h}$.

\section{Acknowledgements}

We thank both DSM Research and EPSRC (EP/G007950/1) for post-doctoral support of MS and the same sponsors for an EPSRC CASE PhD studentship for ERJ. SPA acknowledges receipt of an ERC Advanced Investigator grant (PISA 320372). Dr Dominik Konkolewicz and Dr Ben MacCreath (Agilent) are thanked for their assistance with the recalculation of the deconvoluted GPC data.

\section{Notes and references}

1 D. E. Discher and A. Eisenberg, Science, 2002, 297, 967.

2 M. W. Matsen and F. S. Bates, Macromolecules, 1996, 29, 1091.

3 J. A. Balmer, S. P. Armes, P. W. Fowler, T. Tarnai, Z. Gáspár, K. A. Murray and N. S. J. Williams, Langmuir, 2009, 25, 5339.

4 J. N. Israelachvilli, D. J. Mitchell and B. W. Ninham, J. Chem. Soc., Faraday Trans. 2, 1976, 72, 1525.

5 L. Zhang and A. Eisenberg, Science, 1995, 268, 1728.

6 Y.-Y. Won, H. T. Davis and F. S. Bates, Science, 1999, 283, 960.

7 Z. Li, E. Kesselman, Y. Talmon, M. A. Hillmyer and T. P. Lodge, Science, 2004, 306, 98.

8 H. Cui, Z. Chen, S. Zhong, K. L. Wooley and D. J. Pochan, Science, 2007, 317, 647.

9 X. Wang, G. Guerin, H. Wang, Y. Wang, I. Manners and M. A. Winnik, Science, 2007, 317, 644.

10 D. J. Pochan, Z. Chen, H. Cui, K. Hales, K. Qi and K. L. Wooley, Science, 2004, 306, 94.

11 S. Jain and F. S. Bates, Science, 2003, 300, 460.

12 D. A. Christian, A. Tian, W. G. Ellenbroek, I. Levental, K. Rajagopal, P. A. Janmey, A. J. Liu, T. Baumgart and D. E. Discher, Nat. Mater., 2009, 8, 843.

13 M. Massignani, C. LoPresti, A. Blanazs, J. Madsen, S. P. Armes, A. L. Lewis and G. Battaglia, Small, 2009, 5, 2424.

14 J. R. Howse, R. A. L. Jones, G. Battaglia, R. E. Ducker, G. J. Leggett and A. J. Ryan, Nat. Mater., 2009, 8, 507.

15 R. C. Hayward and D. J. Pochan, Macromolecules, 2010, 43, 3577.

16 A. C. Arsenault, D. A. Rider, N. Tetreault, J. I. L. Chen, N. Coombs, G. A. Ozin and I. Manners, J. Am. Chem. Soc., 2005, 127, 9954.

17 J. Y. Cheng, A. M. Mayes and C. A. Ross, Nat. Mater., 2004, 3, 823. 18 R. Savic, L. Luo, A. Eisenberg and D. Maysinger, Science, 2003, 300, 615.

19 P. Cotanda and R. K. O’Reilly, Chem. Commun., 2012, 48, 10280.

20 Z. An, Q. Shi, W. Tang, C.-K. Tsung, C. J. Hawker and G. D. Stucky, J. Am. Chem. Soc., 2007, 129, 14493.

21 B. Charleux, G. Delaittre, J. Rieger and F. D'Agosto, Macromolecules, 2012, 45, 6753.

22 J.-T. Sun, C.-Y. Hong and C.-Y. Pan, Soft Matter, 2012, 8, 7753. 
23 A. Blanazs, J. Madsen, G. Battaglia, A. J. Ryan and S. P. Armes, J. Am. Chem. Soc., 2011, 133, 16581.

24 A. Blanazs, A. J. Ryan and S. P. Armes, Macromolecules, 2012, 45, 5099.

25 P. Chambon, A. Blanazs, G. Battaglia and S. P. Armes, Macromolecules, 2012, 45, 5081.

26 E. R. Jones, M. Semsarilar, A. Blanazs and S. P. Armes, Macromolecules, 2012, 45, 5091.

27 Y. Li and S. P. Armes, Angew. Chem., Int. Ed., 2010, 49, 4042.

28 M. Semsarilar, E. R. Jones, A. Blanazs and S. P. Armes, Adv. Mater., 2012, 24, 3378.

29 M. Semsarilar, V. Ladmiral, A. Blanazs and S. P. Armes, Langmuir, 2011, 28, 914.

30 S. Sugihara, A. Blanazs, S. P. Armes, A. J. Ryan and A. L. Lewis, J. Am. Chem. Soc., 2011, 133, 15707.

31 J. Rosselgong, A. Blanazs, P. Chambon, M. Williams, M. Semsarilar, J. Madsen, G. Battaglia and S. P. Armes, ACS Macro Lett., 2012, 1, 1041.

32 M. F. Cunningham, Prog. Polym. Sci., 2008, 33, 365.

33 M. J. Monteiro and M. F. Cunningham, Macromolecules, 2012, 45, 4939.

34 S. Boissé, J. Rieger, K. Belal, A. Di-Cicco, P. Beaunier, M.-H. Li and B. Charleux, Chem. Commun., 2010, 46, 1950.

35 I. Chaduc, W. Zhang, J. Rieger, M. Lansalot, F. D’Agosto and B. Charleux, Macromol. Rapid Commun., 2011, 32, 1270.

36 W. Zhang, F. D'Agosto, O. Boyron, J. Rieger and B. Charleux, Macromolecules, 2011, 44, 7584.

37 W. Zhang, F. D’Agosto, O. Boyron, J. Rieger and B. Charleux, Macromolecules, 2012, 45, 4075.

38 X. Zhang, S. Boissé, C. Bui, P.-A. Albouy, A. Brulet, M.-H. Li, J. Rieger and B. Charleux, Soft Matter, 2012, 8, 1130.

39 X. Zhang, S. Boissé, W. Zhang, P. Beaunier, F. D’Agosto, J. Rieger and B. Charleux, Macromolecules, 2011, 44, 4149.

40 G. Liu, Q. Qiu, W. Shen and Z. S. An, Macromolecules, 2011, 44, 5237.

41 W. Shen, Y. Chang, G. Liu, H. Wang, A. Cao and Z. S. An, Macromolecules, 2011, 44, 2524.

42 G. Liu, Q. Qiu and Z. An, Polym. Chem., 2012, 3, 504.

43 X. Zhang, J. Rieger and B. Charleux, Polym. Chem., 2012, 3, 1502.

44 W. Cai, W. Wan, C. Hong, C. Huang and C.-Y. Pan, Soft Matter, 2010, 6, 5554.

45 W.-D. He, X.-L. Sun, W.-M. Wan and C.-Y. Pan, Macromolecules, 2011, 44, 3358.

46 C.-Q. Huang and C.-Y. Pan, Polymer, 2010, 51, 5115.

47 W.-M. Wan and C.-Y. Pan, Polym. Chem., 2010, 1, 1475.

48 M. Yokota, Y. Miwa, H. Ajiro and M. Akashi, Polym. J., 2012, 44, 301.

49 S. Iwasaki, Z. Satoh, H. Shafiee, A. Tagaya and Y. Koike, Polymer, 2012, 53, 3287.

50 L. Nurmi, H. Peng, J. Seppala, D. M. Haddleton, I. Blakey and A. K. Whittaker, Polym. Chem., 2010, 1, 1039.
51 H. Lu, F. Su, Q. Mei, Y. Tian, W. Tian, R. H. Johnson and D. R. Meldrum, J. Mater. Chem., 2012, 22, 9890.

52 Y. K. Chong, T. P. T. Le, G. Moad, E. Rizzardo and S. H. Thang, Macromolecules, 1999, 32, 2071.

53 M. Mertoglu, A. Laschewsky, K. Skrabania and C. Wieland, Macromolecules, 2005, 38, 3601.

54 G. Moad, E. Rizzardo and S. H. Thang, Aust. J. Chem., 2006, 59, 669.

55 A. B. Lowe and C. L. McCormick, Prog. Polym. Sci., 2007, 32, 283.

56 S. Perrier and P. Takolpuckdee, J. Polym. Sci., Part A: Polym. Chem., 2005, 43, 5347.

57 Polymer Handbook, ed. J. Brandrup and E. H. Immergut, Interscience, 1966.

58 C. L. McCormick and A. B. Lowe, Acc. Chem. Res., 2004, 37, 312.

59 D. B. Thomas, A. J. Convertine, R. D. Hester, A. B. Lowe and C. L. McCormick, Macromolecules, 2004, 37, 1735.

60 J.-F. Baussard, J.-L. Habib-Jiwan, A. Laschewsky, M. Mertoglu and J. Storsberg, Polymer, 2004, 45, 3615.

61 K. Skrabania, A. Miasnikova, A. M. Bivigou-Koumba, D. Zehm and A. Laschewsky, Polym. Chem., 2011, 2, 2074.

62 One reviewer of this manuscript suggested that THF may be an inappropriate choice of solvent for the control experiments in which TFEMA is polymerized in homogeneous solution. This is because this solvent is known to be susceptible to the gradual build-up of appreciable peroxide levels over time. In addition, others have shown that use of THF as a solvent for certain RAFT syntheses can result in significant oxidative degradation of the RAFT chain-ends (see T. Gruendling, R. Pickford, M. Guilhaus and C. Barner-Kowollik, J. Polym. Sci., Part A: Polym. Chem., 2008, 46, 7447). However, we used a fresh bottle of THF $(99.8 \%$ purity) for all solution polymerizations, which should minimize this problem. Moreover, the above earlier study focused on a dithiobenzoate-based RAFT agent, whereas our work involved a trithiocarbonate-based RAFT agent, which is usually considered to be more stable. Finally, similar changes in UV-visible absorption spectra to those shown in Fig. 7 were also observed when using DMF instead of THF (data not shown), which suggests that the enhanced RAFT chain-end degradation found for the solution polymerizations described herein is not simply due to the peroxide levels in the latter solvent.

63 G. Moad, E. Rizzardo and S. H. Thang, Polymer, 2008, 49, 1079.

64 G. Moad, E. Rizzardo and S. H. Thang, Acc. Chem. Res., 2008, 41, 1133.

65 R. McHale, J. P. Patterson, P. B. Zetterlund and R. K. O’Reilly, Nat. Chem., 2012, 4, 491.

66 P. B. Zetterlund, Polym. Chem., 2011, 2, 534. 